\title{
Resilience
}

\section{From Policy Responses to Resilient Policy Systems}

\section{JOHN MCDERMOTT, DANIELLE RESNICK, AND NICHOLA NAYLOR}

John McDermott is director of the CGIAR Research Program on Agriculture for Nutrition and Health, International Food Policy Research Institute (IFPRI), Washington, DC. Danielle Resnick is a senior research fellow, Development Strategy and Governance Division, IFPRI,

Washington, DC. Nichola Naylor is a research fellow, London School of Hygiene and Tropical Medicine, London.

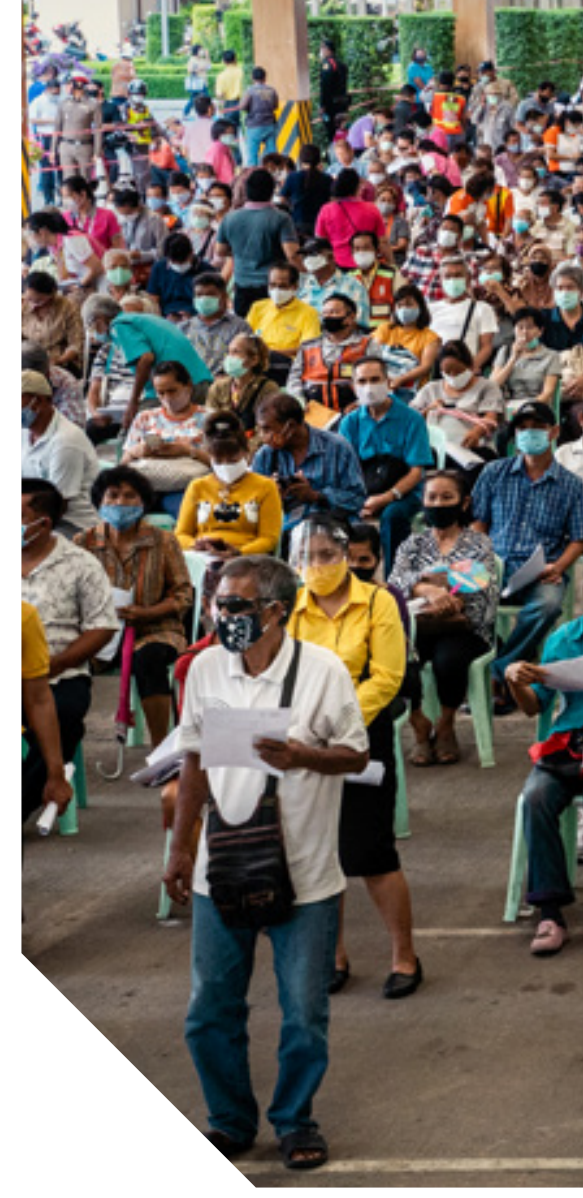

\section{KEY MESSAGES}

- Policy responses across countries have followed a similar course - beginning with lockdowns and health measures, then shifting toward fiscal, monetary, and social protection interventions - as the pandemic progressed.

- Policymakers must balance critical trade-offs among policy actions and spending priorities for health, food systems, and economies. This requires a multisectoral perspective and clearly defined values.

- Rapid policy actions must build on existing systems; therefore, the quality of established policy and delivery systems is an important determinant of success.

- Three broad features of policy systems - adaptability, coherence and coordination, and efficacy - contribute to the success of public policies.

- Policy system efficacy requires state capacity for administrative efficiency and appropriate enforcement, as well as citizen trust in government.

- Rolling out and scaling up innovations during crises depends on an underlying business environment that enables decision-making and experimentation in partnership with the private sector.

\section{RECOMMENDATIONS}

- Build effective and resilient policy systems and programs to enable decision-makers to respond to future crises in an informed, timely, and cohesive manner and in a way that builds credibility and confidence among citizens.

- Increase understanding of the interplay of health, economic, and social policy actions, gather more data, and review experience to aid decision-making.

- Develop robust public systems for the poor and vulnerable, such as social protection, nutrition, and education programs that can be quickly scaled up and adapted.

- Support an enabling business environment to spur private innovation in food systems, particularly by small and medium enterprises.

- Develop processes for policy coordination and increase capacity of policymakers to work across multiple sectors, vertically, horizontally, and temporally, to address crises and support transformation toward more resilient food systems. 
of vaccination plans. The rebuilding phase should develop longer-term policies and strategies for sustainability and resilience of food, economic, and health systems. However, the actual pattern of both disease and policy evolution is more complicated, with substantial overlap in implementation of these phases, which is not surprising given the unpredictable nature and persistence of this coronavirus. Most countries are still in the response and recovery phases.

In the initial emergency response phase, rapid action was essential to address the fear and pessimism of individuals and institutions, and included lockdowns to limit deaths and avoid overwhelming health systems, as well as economic stimulus measures to address the loss of incomes. Domestic and international financial resources were mobilized quickly in many countries. While the hope was that these emergency responses would eliminate or greatly reduce coronavirus infections, this effort failed in most countries. Infection spread has followed some predictable patterns linked to population density, demography, and seasons, but these are invariably complicated by hard-to-predict waves of infection and "super-spreading" behaviors and events. ${ }^{2}$ As a result, initial emergency health responses were adapted during subsequent disease waves.
As economic, food security, and social challenges of the pandemic became apparent, countries began to adopt short-term economic and financial measures to facilitate a medium- and long-term pandemic response that would spur recovery and promote resilience. Social protection and other programs to support vulnerable populations were expanded or put in place over the course of the year. Near the end of 2020, attention began shifting to recovery measures, such as planning for vaccinations.

The unprecedented nature of the pandemic has meant that policymakers had little evidence to draw on for choosing policies during initial stages. Impressive efforts started early and continue to collect, collate, and analyze a wide range of pandemic-related policy actions taken by different countries; these are making a wealth of information available to policymakers. One widely used policy aggregator is the Oxford Global Directory for COVID Policy Trackers and Surveys. ${ }^{3}$ Many of these efforts aim to compare and contrast country policy experiences, but such comparisons are challenging given the range of country contexts, resources, and capacities for responding to the crisis. ${ }^{4}$ In general, policymakers relied on broad principles and guidance, which they adapted to the evolving national and subnational conditions they faced,

\section{BOX 1 COUNTRY CONTEXT, CORONAVIRUS INFECTIONS, AND POLICY RESPONSES}

The policy actions taken by the countries included in Figure 1 reflect their unique circumstances, infection dynamics, and societal disruptions. Bangladesh, a densely populated, lower-middle-income country, faced an acute double challenge of coronavirus transmission and loss of livelihoods for its many poor day-laborers. Strict lockdowns were not accepted and could not be enforced, but COVID-19 cases remained relatively stable. Without large-scale social protection infrastructure, financial stimulus was the main policy response. Neighboring Myanmar was able to implement an initial temporary lockdown that limited cases, but it had to be reinstated in a major city when a new wave of infection occurred. Egypt's main challenges were the loss of tourism and remittance incomes and its effect on households and businesses. Strict lockdowns were avoided and COVID-19 cases remained relatively controlled, allowing policymakers to focus on support to households and businesses and to consider longer-term measures to enable efficiency, effectiveness, and competitiveness of food systems (see Box 2). In Rwanda, social compliance with rules and regulations is high, and the country managed to control infections with targeted lockdowns and move quickly to economic recovery. Zambia also used targeted lockdowns while implementing social protection programs; however, the country faced a marked second infection wave late in 2020, and a government debt crisis will limit subsequent stimulus. Finally, Honduras implemented strict lockdown measures but other concurrent crises, including hurricanes Eta and lota, overwhelmed an already fragile healthcare system. Emergency social measures focused on household food supplies. See also comparable figures in the Regional Developments section of this report. 


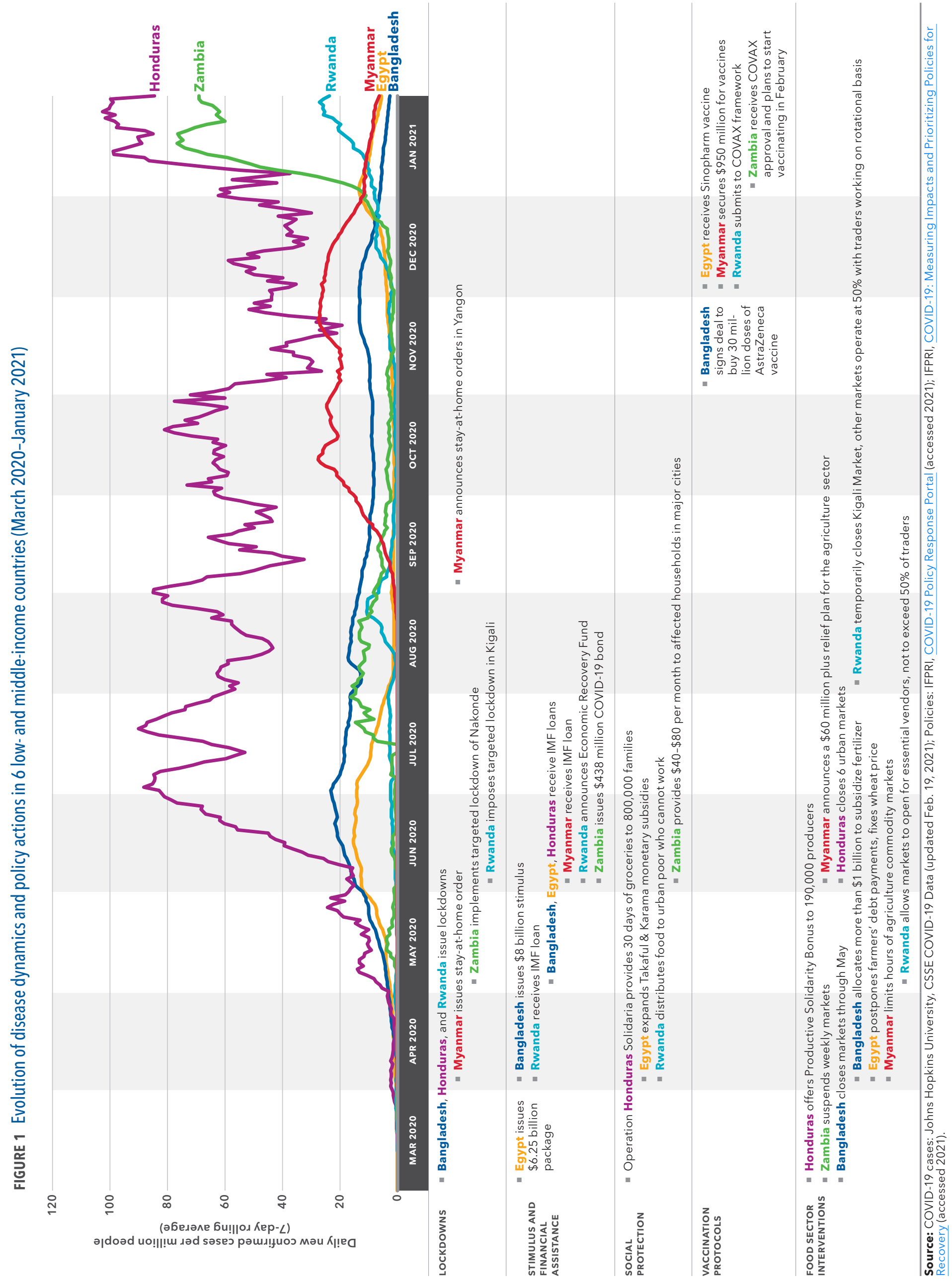




\section{BOX 2 POLICY PRIORITIES IN EGYPT}

\section{Dr. Ahmed Kamaly, Deputy Minister, Egyptian Ministry of Planning and Economic Development}

At the outset of the pandemic, the Ministry of Planning and Economic Development had to assess the impacts on various components of GDP, including employment, poverty, and the balance of payments. To gain a comprehensive assessment, we consulted with other ministries, business associations, civil society, and academia. We tried as much as possible to rely on evidence-based policymaking because with this type of crisis, and the huge uncertainty around it, nobody knew what was going to happen. So, we turned to many sources of information, including the UN agencies, IFPRI, IMF, World Bank, and other entities.

Although we had many priorities, there were a few areas we felt needed to be especially targeted. For instance, at the beginning of the crisis, we recognized that informal workers were going to be badly affected and therefore, we provided unemployment benefits to these individuals, which we continue to do. We further anticipated a big hit in the tourism and manufacturing sectors and tried to identify policies to support those sectors. We also aimed to support ICT-based solutions because ICT is both the future and essential for mitigating the negative impacts of COVID-19 on other sectors. In addition, we provided some financial support to help businesses cover their operational expenditures and thereby prevent bankruptcies.

While it is important to track the costs versus benefits of different policy actions to determine whether to continue them, this is very difficult to do in response to COVID-19 because we adopted bundles of policies simultaneously and identifying their impacts will take time. However, the crisis has also taught us that with shocks, there are some opportunities. So, we are now looking at how we should reform our business climate and increase the productivity of our supply chains to be more competitive when this storm passes. In addition, the pandemic has highlighted that while social protection is key for helping the poor, poverty is only a symptom of the root problem, which is the lack of decent jobs in Egypt.

Note: These are personal observations and should not be construed as the official positions of the ministry.

including national public health, economic, and sociopolitical contexts. Our interviews with policymakers reflect the different perspectives they considered in the short and medium terms (Boxes 2 and 3).

Given the urgency of the pandemic, implementation of policy actions had to build on existing systems and programs; thus the quality of the established policy and delivery systems was an important determinant of success. For example, early policy responses often expanded and adapted existing social protection programs (Chapter 5). This was effective in Ethiopia, where the well-established Productive Safety Net Program was expanded. ${ }^{5}$ While these social protection programs are relatively common in middle-income countries, there were few such programs to build on in many low-income countries. Also, the limited fiscal capacity of low- and lower-middle-income countries has meant that, after the initial emergency actions, critical decisions and trade-offs had to be made, for example, selecting which people and places to target with the limited funding available. In some countries, such as Zambia, debt burdens may preclude any additional government action.

What has this meant for food systems? In most countries, established agrifood policies and objectives supported a long-term food system transformation agenda focused on job creation and healthier and sustainable diets (as described in IFPRI's 2020 Global Food Policy Report). ${ }^{6}$ However, implementation lags and new issues that surfaced during the pandemic should lead to adaptations to better support recovery and build resilience. While primary agricultural production was often considered a priority sector for ensuring food security and so exempted from pandemic restrictions, food marketing and services usually were not. In the absence of targeted policies to balance restrictions on food markets and services with longer opening hours and more space, these operations suffered extensive disruption. ${ }^{7}$ In Nigeria, for example, primary production declined by 15 percent 


\section{BOX 3 POLICY COORDINATION AND OPPORTUNITIES IN NIGERIA}

\section{Dr. Andrew Kwasari, Senior Special Assistant to the President on Agriculture and Head of the Project for Agriculture Coordination and Execution (PACE), Office of the Vice President of Nigeria}

Coordination is a very important consideration for the Nigerian government as it confronts COVID-19. At the beginning of the pandemic, the President of Nigeria constituted a committee chaired by the Vice President. This committee included five sectors viewed as key for the country: agriculture, trade and investment, works and housing, minerals, and petroleum and gas. Along with the minsters for these sectors and technical support staff, an Economic Sustainability Plan (ESP) was developed that became the main framework for coordinated policy action. During the process of developing the ESP, the Vice President consulted with the Nigeria Governors' Forum. We selected one governor per geopolitical zone who then consulted with other governors in their zones, and then we had a series of virtual meetings at every stage of the ESP to ensure broad stakeholder support. We also consulted widely with commodity associations, regionally based think tanks, and captains of industry in agriculture, such as Aliko Dangote.

We realized very early on, from both our own analyses and those of international organizations and consulting firms, that agriculture could be badly affected by the coronavirus. We anticipated that there would be food shortages globally as countries prioritized their own food security over international food trade. At the same time, our main source of foreign exchange - oil and gas - was hit by low demand due to shuttered industries and a rift within OPEC (Organization of the Petroleum Exporting Countries). So, we realized that due to reduced potential for importing food, it was critical to protect our national food system and our rural populations from the virus. We decided to focus on support for local production by providing farmers with needed inputs and finance and solving logistical barriers along the food chain. For example, governors originally were stopping trucks at their state borders, but the President, Vice President, and the Federal Minister of Agriculture and Rural Development intervened to allow trucks with farm inputs and food to continue their operations. In doing so, we collaborated with the National Union of Transport Workers to ensure they confirmed trucks only with those materials could cross state borders.

Despite the economic and health costs, there have been some real opportunities due to COVID-19. For instance, we realized that if we were going to make agriculture more resilient and help entrepreneurs view agriculture as a business, we needed a database of farmers to identify where they are located and how best to support them. With financing from the federal government, technical staff from the states, and the development of the Nigerian Agricultural Assets Survey App, we trained young enumerators across the country to register farmers, collect their farm GIS information, and gather other information about the commodities they cultivate. By the end of 2020, we had 5.4 million farmers registered. We subsequently worked with a variety of commercial banks, and now 2.3 million of these farmers are financially included in the banking system. By creating this digital database of farmers, we also now have ways of depositing subsidy transfers for agricultural inputs into their bank accounts. In this way, we have leveraged different technologies to strengthen the food system to face future crises.

Note: These are personal observations and should not be construed as the official positions of the government.

and food services by 80 percent. ${ }^{8}$ Trade and marketing of cereals and other staples were relatively unaffected, although some standard shock responses, such as restricting cereal exports (for example, rice from Viet Nam), were implemented temporarily. Supply chains for perishable products often suffered the greatest disruptions, in part because of concerns over the safety of animal-sourced foods. ${ }^{9}$ On the positive side, these disruptions also accelerated private sector innovation, particularly for digital tracking and service delivery, that can contribute to food system transformation (Chapter 6). ${ }^{10}$

\section{THE CHALLENGE OF BALANCING PUBLIC HEALTH, FOOD SYSTEM, AND ECONOMIC IMPACTS}

As the pandemic led to widespread disruptions across the whole of society, the typical standalone sectoral policy responses used to address financial or food-supply crises or disease outbreaks, such as SARS and Ebola, were inadequate - more cross-governmental and cross-sectoral policy design and implementation were needed. ${ }^{11}$ The health and economic trade-offs associated with pandemic policy 
decisions are not simple. ${ }^{12}$ On the health side, policies are needed to prevent widespread illness and death, high healthcare system costs, and subsequent lost productivity. Uncontrolled virus spread can overwhelm health systems, with estimated costs ranging from US $\$ 50$ per capita in low-income countries to $\$ 84$ in upper-middle-income countries. ${ }^{13}$ Thirty-day nationwide lockdowns (which aim to reduce transmission, illness and deaths, and healthcare needs) of various stringencies are thought to reduce the healthcare burden substantially, from an estimated average of $\$ 5.2$ billion down to $\$ 4.7$ billion per country. ${ }^{14}$ However, lockdowns may also impose indirect health losses through reduced access to care and a heavy economic burden, especially on the most vulnerable in society, through lost income (Chapter 5$)^{15}$

On the economic side, the pandemic together with the responses designed to slow its spread have caused a global recession. Economic growth is expected to have fallen by 0.9 percent in 2020 for low-income countries as a result of the pandemic. ${ }^{16}$ This economic contraction is linked to multiple disruptions, both domestic and international, ${ }^{17}$ which have sparked great concern about the pandemic's trajectory, the long-term impacts on growth and other economic indicators, and the future economic landscape. ${ }^{18}$ Thus the implications of coronavirus control and eradication extend beyond boundaries, requiring a global solution.

To illustrate the association between lockdowns and reduced economic activity, Figure 2 plots a snapshot of the stringency of lockdown and other closure measures for 14 countries in the early months of the pandemic against the declines in national GDP and agricultural GDP (relative to the same period in 2019). In general, more stringent lockdowns are associated with greater decreases in GDP (with some exceptions, such as Mali and Malawi). However, the decline is not uniform; lockdowns have different impacts on different sectors. The association is generally weaker for agricultural GDP, likely reflecting the fact that agriculture was exempted from some restrictions. For low-income countries, primary agriculture, which suffered less disruption than value-added food sectors beyond the farm, is a much greater component of overall food sector GDP. Thus, lockdowns would be expected to have less impact on their agricultural
GDP. For higher-income countries, where a greater share of food sector GDP is generated beyond primary agriculture, lockdowns may have a greater impact. They will need to be considered more carefully in the policymaking process in order to better understand the trade-offs being made in fiscal decisions and in burden-sharing as food systems transform.

The distribution of the disease burden and the economic burden across social groups within countries is also an important concern for policymakers. For example, in Bangladesh, a strict lockdown was not enforceable for poor day-laborers. Throughout low- and middle-income countries, the poor and other vulnerable populations bear the brunt of the economic impacts of response policies and are also less able to avoid contracting the virus. ${ }^{19}$ For instance, disproportionately more people in lower socioeconomic quintiles in Pakistan and the Philippines experienced income loss and were unable to socially distance in lockdown simulation exercises. ${ }^{20}$

To mitigate the health and economic impacts on vulnerable populations, a World Bank policy framework advocates for a fast, hard, and short response to stop the transmission of the virus, followed by substantial fiscal and monetary stimulus in a recovery phase to mitigate the economic impacts. ${ }^{21}$ However, as discussed above, LMICs may not have capacity for the fiscal and monetary stimulus necessary to adequately support vulnerable populations. It is estimated that additional donor aid of approximately $\$ 5$ billion (about half the total required) is needed to meet this shortfall and prevent millions from being pushed into poverty and food insecurity. ${ }^{22}$ At the same time, the anticipated long-lasting economic effects of the pandemic suggest that the public may not be satisfied with just a short-term response and could expect mitigation measures to continue for the foreseeable future.

Public health policymakers are already attempting to bring economic, social, and health perspectives into their planning for recovery and future resilience to ensure well-balanced policies. Box 4 uses the example of vaccine distribution to illustrate some approaches to prioritizing public health interventions, considering different combinations of health, social, and economic parameters. With growing evidence on the role of household characteristics, behavior, capacity, and resilience in shaping outcomes during pandemics, 


\section{FIGURE 2 Lockdown stringency and change in GDP and agricultural GDP}

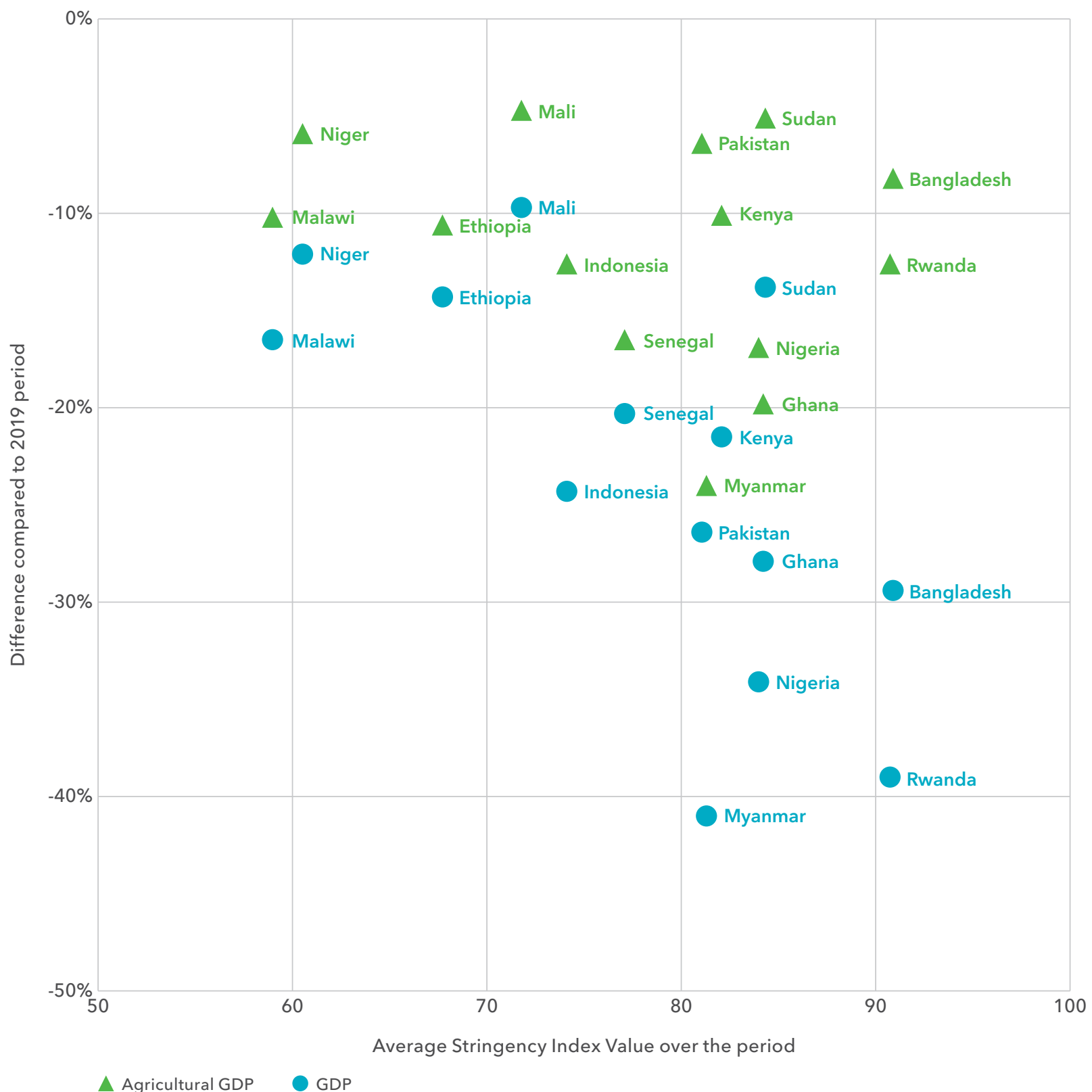

Source: Stringency data are from H. Thomas et al., Oxford's COVID-19 Government Response Tracker, Blavatnik School of Government (www.bsg.ox.ac.uk/covidtracker, accessed Feb. 8, 2021), and averaged over the same time period as the snapshot analysis of GDP and agricultureand food-related GDP performed by IFPRI (see COVID-19: Measuring Impacts and Priotizing Policies for Recovery).

Note: Stringency is measured by the Oxford Stringency Index (national level), which is a composite measure of nine policy response indicators such as "restrictions on internal movement." Declines in GDP (gross domestic product) and agricultural GDP are relative to the average corresponding values in the same period in 2019 (for example, if lockdown occurred in March-April in 2020, the GDP for that period was compared to that of March-April 2019). The dates of the analyses vary by country due to different start and end dates of national policies, and dates of latest available economic analyses. GDP snapshot periods run from a few weeks to a few months between February and June 2020. Some countries included had a full lockdown, others had partial lockdowns or curfews, which is reflected in the stringency index. 


\section{BOX 4 INCORPORATING ECONOMIC AND SOCIAL PARAMETERS INTO HEALTH DECISIONS: TARGETING VACCINE DISTRIBUTION}

In deciding whether to implement a national lockdown, what testing protocols to implement, or whom to vaccinate, a country is making value choices about what objectives to prioritize and how to distribute the burden of COVID-19 and the costs and benefits of associated policies across the population. Taking different ethical and social justice perspectives will lead to prioritizing different policies and populations. Here we explore how different perspectives can shape vaccine distribution.

ONE HEALTH PERSPECTIVE: Different decision-makers across the One Health ${ }^{a}$ system may have different, and sometimes even competing, perspectives and objectives that emphasize human health, animal health and use, agriculture, environment, and other government goals. ${ }^{\text {b }}$ For example, national treasuries may be interested in minimizing short-term economic GDP impact while environmental agencies may be interested in preventing longer-term pollution. Explicitly defining and understanding these differences early can allow for more effective discussion, selection, and implementation of COVID-19 vaccination strategies.

SOCIAL JUSTICE PERSPECTIVE: Deciding whom to vaccinate first presents an ethical dilemma when vaccines are scarce. Should countries seek to maximize benefits across the whole population (which usually means favoring the young, who have more years of life ahead of them than the elderly) or seek to minimize inequality and inequity (by targeting those in greatest need or with the least access)? ${ }^{c}$ By clarifying the aggregate costs in terms of years of life lost or the aggregate GDP impact of specific vaccination policies, along with the distribution of these impacts across specific subpopulations, both overall benefits and equity impacts can be considered in a structured way. ${ }^{d}$ For example, for infectious diseases such as COVID-19, poorer populations may be more severely affected since they have limited capacity to socially distance and only obtain income through in-person labor. Thus vaccinating such frontline poor workers mitigates multiple risks and has multiple benefits.

The World Health Organization SAGE Working Group on COVID-19 vaccines proposes that policymakers allow for "the integration of explicit values with evolving scientific and economic evidence." ${ }^{\text {e }}$ Recommended values to consider include human wellbeing, equal respect, global equity, national equity, reciprocity, and legitimacy. Building on this, scenarios for various levels of vaccine availability are proposed: ${ }^{f}$

- $11-20 \%$ of national population: Sociodemographic groups at significantly higher risk of severe disease or death should receive the vaccine.

- $21-50 \%$ of national population: Extend vaccination to essential workers outside the health and education sectors, including agriculture and food workers and groups at elevated risk of acquiring and transmitting infection because they are unable to effectively physically distance.

National prioritization decisions may select different priority populations based on the levels of risk of infection or harm (in all senses described in this section) and on the preference given to different values within that country.

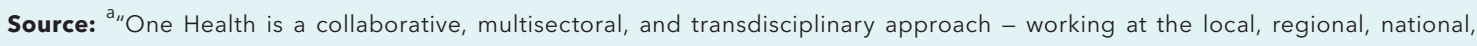
and global levels - with the goal of achieving optimal health outcomes recognizing the interconnection between people, animals, plants, and their shared environment" (CDC, accessed Feb. 2021, https://www.cdc.gov/onehealth/basics/index.html); ${ }^{b}$ N.R. Naylor et al., "Quantitatively Evaluating the Cross-Sectoral and One Health Impact of Interventions: A Scoping Review and Case Study of Antimicrobial Resistance," One Health 11 (2020): 100194; ' A. Giubilini, J. Savulescu, and D. Wilkinson, "COVID-19 Vaccine: Vaccinate the Young to Protect the Old?," Journal of Law and the Biosciences 7, no. 1 (2020): Isaa050; 'R.E. Glover et al., "A Framework for Identifying and Mitigating the Equity Harms of COVID-19 Policy Interventions," Journal of Clinical Epidemiology 128 (2020); 'World Health Organization, WHO SAGE Values Framework for the Allocation and Prioritization of COVID-19 Vaccination (Geneva: 2020); f S. Omer et al., WHO SAGE Roadmap for Prioritizing Uses of COVID-19 Vaccines in the Context of Limited Supply (Geneva: World Health Organization, 2020). 
along with the expanding pool of disease-transmission models and associated micro- and macroeconomic costing models, 2021 should see more deliberative, evidence-based policymaking that recognizes both the health and economic costs and benefits of policy options for different sectors and social groups. ${ }^{23}$

\section{TOWARD EFFECTIVE AND RESILIENT POLICY SYSTEMS}

As countries move into the recovery and rebuilding stages, we must think about how to make policy systems more effective and resilient. Just like households, policy systems may not be able to withstand shocks and can become constrained by inertia that allows crises to fester or become fragmented by inconsistent and volatile policy initiatives. "Resilient policy systems" therefore refers to systems that enable decision-makers to respond to future crises, single and multiple, in an informed, timely, and cohesive manner and in a way that builds credibility and confidence among citizens. While policy interventions for the current pandemic have varied greatly across countries, certain features of policy systems have been shown to enhance the ability to make rapid and efficacious decisions vis-à-vis health, food systems, and economic livelihoods under conditions of uncertainty. Three broad features of policy systems discussed here - adaptability, coherence and coordination, and efficacy of implementation and enforcement - contribute to both pandemic recovery and future food system transformation.

ADAPTABILITY refers to the ability to modify extant policies to meet new needs, arising either from crises or longer-term changes. In 2020, some countries quickly found innovative ways both to keep markets working while reducing disease transmission and to refocus public healthcare delivery to address pandemic conditions. In Sri Lanka, the century-old weekly tea auction was quickly shifted to an online platform. In East Africa, the Regional Electronic Cargo and Driver Tracking initiative was introduced in response to the pandemic and has facilitated the monitoring of truck drivers for COVID-19, reducing congestion and long wait-times at borders. ${ }^{24}$ In Ghana, drone technology was deployed in rural areas to speed the transport of medical supplies and medical samples for coronavirus testing. ${ }^{25}$ In a number of countries, urban informal workers were incorporated into social protection programs as lockdowns closed markets or reduced trading hours. ${ }^{26}$

Many of these quick adaptations could be successfully implemented under emergency conditions because they built on established programs and relationships between societal groups and the state. The ability to roll out and scale up some innovations during crises depends on an underlying business environment that enables decision-making and experimentation in partnership with the private sector. For example, market innovations and public interventions that rely on information and communications technologies (ICTs), such as digital cash transfers, are more likely to succeed when enabled by established technology governance that encourages innovation and cooperation (without generating new societal costs with respect to privacy, misuse of data-sharing, or inequities in access). ${ }^{27}$ Existing organizations and negotiating processes can also be leveraged quickly for implementation. For instance, one survey found that disbursement of pandemic cash relief payments to informal workers was more effective in cities where active informal workers' associations could help members navigate digital registration platforms. ${ }^{28}$

COHERENCE AND COORDINATION are critical features of policy systems that mitigate against volatility, confusion, and inefficient outcomes. Such coordination has at least three different dimensions: horizontal (across sectors), vertical (across levels of government), and temporal (sequencing of policies). Many countries initially focused on horizontal coordination and established interministerial response teams; from a sample of 33 countries followed by IFPRI's COVID-19 Policy Response Portal, 23 created a COVID-19 taskforce or committee at the start of the pandemic that typically included ministries of health, trade, transport, foreign affairs, police, tourism, finance, and defense. ${ }^{29}$

Vertical coordination was essential given that local governments have played frontline roles during the pandemic. ${ }^{30}$ In Uganda, local governments are responsible for surveillance, behavioral education to limit contagion, enforcing control measures such as curfews, identifying beneficiaries for food distribution, and delivering food aid. ${ }^{31}$ The Kampala Capital City Authority even 
launched an online app for the home-delivery of food from informal vendors whose livelihoods were threatened by the lockdowns. ${ }^{32}$ However, vertical coordination is especially difficult in countries with a high degree of political polarization or with substantial subnational political autonomy, such as federal countries with decentralized health systems. In Nigeria, for example, the governors of two states rejected the testing guidelines of the Presidential Task Force. ${ }^{33}$ In contrast, in Brazil, governors adopted more stringent coronavirus lockdowns and testing and lobbied for quicker vaccination options than the country's president thought necessary. ${ }^{34}$

Temporal coordination has proved exceedingly difficult. In India, the president announced lockdowns with only four hours' notice; but transport systems were unprepared to adapt quickly to meet the needs of millions of migrants returning to rural areas, contributing to a surge in coronavirus infections. ${ }^{35}$ In many African cities, markets were shuttered to assist with social distancing, but this health measure also deprived local governments of needed revenue for water and sanitation management, which are necessary for effective hygiene.

POLICY EFFICACY depends on implementation and credible enforcement and requires state capacity - in terms of both enforcement abilities and administrative efficiency - as well as citizen trust in government. Administrative efficiency depends on the state's technical competency and skills as well as adequate equipment for implementation. This form of capacity is essential to the rapid rollout of any emergency program. In settings of low administrative efficiency, the initial flurry of cash-transfer and food-relief policies was often overshadowed by cases of government corruption, hoarding, or poor accessibility for intended beneficiaries. ${ }^{36}$ Capacities are generally higher where there are more human and financial resources, mechanisms for oversight and accountability, and autonomous public institutions. Such independent public institutions play an especially important role in counterbalancing the substantial political discretion that characterizes rapid responses to crises and can lead to corruption and discrimination that subvert intended policy objectives.

Enforcement needs to be employed with caution. On the one hand, use of the police, military, and surveillance was essential to enforce lockdowns, mask mandates, social distancing, and contact tracing. In Africa, it may be that only Rwanda and South Africa could exert sufficient enforcement capacity to maintain effective lockdowns, and these two countries indeed had among the most stringent lockdowns in the region. ${ }^{37}$ On the other hand, where deployment of enforcement capacity is excessive, it can lead to human rights abuses and stifle the free flow of information, raising questions about government motivations. ${ }^{38}$

Administrative and enforcement capacities may interact. ${ }^{39}$ For instance, in Bangladesh, citizens first complied with the government's lockdown, which involved daily deployments of the military and police to vacate streets after 2 p.m. and close all shops that were subject to the lockdown. But, as the state failed to deliver promised economic assistance and citizens became economically desperate, they began to defy restrictions. In response, the police slowly abandoned the enforcement of lockdowns in May, first tacitly and then officially, despite rising COVID-19 cases. ${ }^{40}$

While balanced but effective state capacity is key for policy efficacy in the short-term, trust in evidence-based information from government, media, or social networks can also be a critical determinant of citizens' compliance with health measures over time. ${ }^{41}$ These processes can be mutually reinforcing; for instance, misinformation exacerbating distrust in scientific evidence related to the coronavirus, or disappointment related to a government's handling of other pandemic-related policies, can create skepticism about the efficacy and safety of coronavirus vaccinations. Many governments now recognize that building trust is fundamental for citizen acceptance of vaccinations and are identifying strategies to do so. ${ }^{42}$ These include engaging respected community leaders, empowering frontline health staff, creating forums for citizens to share concerns, identifying differential concerns across gender and ethnicities, and leveraging multiple information platforms. ${ }^{43}$

\section{POLICIES FOR RESILIENT FOOD SYSTEMS}

In sum, the coronavirus pandemic has been an unusually great challenge for policymakers because it has simultaneously disrupted healthcare systems, food systems, and economic systems. As the impacts of 
COVID-19 have shifted over time, policy responses have likewise evolved based on country contexts and capacities. Balancing health and economic policy actions has been difficult, but there is increasing understanding of their interplay and increasing data and experience available to aid decision-making. Finally, although there is no standard policy framework that all countries can apply, building an effective and resilient policy system characterized by adaptability, coherence and coordination, and efficacy of implementation and enforcement can greatly facilitate policy responses and contribute to the development of more robust and resilient food systems.

Prior to the pandemic, food system transformation was an important development strategy of most LMICs. The pandemic has exposed vulnerabilities in food system transformation strategies - particularly for the key policy objectives of inclusion and jobs for poor and disadvantaged people and more diverse food supplies, especially of nutrient-dense perishables. Two types of longer-term policy actions and investments have proved important during the pandemic. One is support for an enabling business environment, including flexible food trade and markets. These have spurred needed private innovation, particularly by small and medium enterprises. The second is robust public systems for the poor and vulnerable, such as social protection, nutrition, and education programs that can be quickly scaled up and adapted. While there is no common formula to address these challenges across all countries, increased capacity to consider multiple sectors and build effective and resilient policy systems are common elements in supporting more effective food system transformations. 